\title{
An Accurate Computational Model for the Hydration Extent of Atmospherically Relevant Carbonyls on Aqueous Atmospheric Particles
}

\author{
Matthew J. Elrod,* Jane A. Sedlak, and He Ren \\ Department of Chemistry and Biochemistry, Oberlin College, Oberlin, Ohio, 44074 USA \\ Email: mjelrod@oberlin.edu
}

Contents: thermodynamics parameters (4 pages, 1 table)

Table S1

References 


\begin{tabular}{|c|c|c|c|c|c|c|c|c|}
\hline \multirow[t]{2}{*}{ system } & \multicolumn{3}{|c|}{ MG2MS } & \multicolumn{3}{|c|}{ van't Hoff adjusted } & \multirow{2}{*}{$\begin{array}{c}\Delta_{\mathrm{r}} \mathrm{G}^{\exp } \text { adjusted } \\
\Delta_{\mathrm{r}} \mathrm{G}^{\mathrm{o}}\end{array}$} & \multirow{2}{*}{$\begin{array}{c}\text { experimental } \\
\Delta_{\mathrm{r}} \mathrm{G}^{\mathrm{o}}\end{array}$} \\
\hline & $\Delta_{\mathrm{r}} \mathrm{H}^{\mathrm{o}}$ & $\Delta_{\mathrm{r}} \mathrm{S}^{\mathrm{o}}$ & $\Delta_{\mathrm{r}} \mathrm{G}^{\mathrm{o}}$ & $\Delta_{\mathrm{r}} \mathrm{H}^{\mathrm{o}}$ & $\Delta_{\mathrm{r}} \mathrm{S}^{\mathrm{o}}$ & $\Delta_{\mathrm{r}} \mathrm{G}^{\mathrm{o}}$ & & \\
\hline formaldehyde & -9.4 & -36 & 1.4 & -9.8 & -17 & -4.7 & -4.0 & $-4.6^{1}$ \\
\hline sulfato formaldehyde ion & 0.2 & -41 & 12.5 & -0.2 & -22 & 6.4 & 7.1 & \\
\hline acetaldehyde & -6.0 & -39 & 5.4 & -6.4 & -19 & -0.7 & 0.0 & $-0.2^{\mathrm{a}}$ \\
\hline glycolaldehyde & -7.5 & -39 & 4.0 & -7.9 & -19 & -2.2 & -1.4 & $-1.4^{2}$ \\
\hline 2-sulfato acetaldehyde ion & -8.3 & -40 & 3.7 & -8.7 & -21 & -2.4 & -1.7 & \\
\hline glyoxal & -9.6 & -38 & 1.6 & -10.0 & -18 & -4.6 & -3.8 & \\
\hline monohydrated glyoxal & -9.2 & -38 & 2.0 & -9.6 & -18 & -4.1 & -3.4 & \\
\hline glyoxylic acid & -11.4 & -38 & 0.0 & -11.8 & -19 & -6.1 & -5.4 & $-3.4^{3}$ \\
\hline propionaldehyde & -6.0 & -39 & 5.5 & -6.4 & -19 & -0.6 & 0.1 & $-0.1^{a}$ \\
\hline acetone & -0.4 & -42 & 12.2 & -0.8 & -23 & 6.1 & 6.8 & $3.7^{\mathrm{a}}$ \\
\hline hydroxyacetone & -5.1 & -41 & 7.2 & -5.5 & -22 & 1.1 & 1.8 & $2.3^{\mathrm{a}}$ \\
\hline fluoroacetone & -5.1 & -41 & 7.1 & -5.5 & -22 & 1.0 & 1.7 & $1.1^{4}$ \\
\hline chloroacetone & -6.5 & -42 & 6.1 & -6.9 & -23 & 0.0 & 0.7 & $1.4^{4}$ \\
\hline sulfatoacetone ion & -6.2 & -42 & 6.4 & -6.6 & -23 & 0.2 & 1.0 & $1.5^{\mathrm{a}}$ \\
\hline nitratoacetone & -7.5 & -45 & 6.0 & -7.9 & -26 & -0.2 & 0.6 & \\
\hline 1,1-dichloroacetone & -8.1 & -43 & 4.7 & -8.5 & -24 & -1.4 & -0.7 & $-0.6^{4}$ \\
\hline dihydroxyacetone & -7.8 & -42 & 4.7 & -8.2 & -23 & -1.5 & -0.7 & $-0.4^{a}$ \\
\hline 1,3-dichloroacetone & -8.9 & -43 & 4.1 & -9.3 & -24 & -2.1 & -1.3 & $-0.8^{4}$ \\
\hline 3-sulfato-1-hydroxyacetone ion & -6.6 & -41 & 5.5 & -7.0 & -21 & -0.7 & 0.1 & $0.0^{\mathrm{a}}$ \\
\hline lactaldehyde & -7.8 & -39 & 3.7 & -8.2 & -20 & -2.4 & -1.7 & $-1.9^{5}$ \\
\hline methylglyoxal (1) & -9.1 & -38 & 2.3 & -9.5 & -19 & -3.8 & -3.1 & \\
\hline methylglyoxal (2) & -7.6 & -40 & 4.4 & -8.0 & -21 & -1.7 & -1.0 & \\
\hline monohydrated (1) methylglyoxal & -6.9 & -41 & 5.4 & -7.3 & -22 & -0.8 & 0.0 & $0.2^{6}$ \\
\hline pyruvic acid & -6.5 & -40 & 5.3 & -6.9 & -21 & -0.8 & -0.1 & $-0.4^{\mathrm{a}}$ \\
\hline butanal & -6.0 & -39 & 5.5 & -6.4 & -19 & -0.6 & 0.1 & $0.2^{7}$ \\
\hline 2-butanone & -2.3 & -43 & 10.6 & -2.7 & -24 & 4.5 & 5.2 & \\
\hline 2,3-butanedione & -4.7 & -39 & 7.0 & -5.1 & -20 & 0.9 & 1.6 & $-0.4^{4}$ \\
\hline 1-sulfato-2,3-butanedione ion & -6.6 & -44 & 6.4 & -7.0 & -24 & 0.3 & 1.0 & \\
\hline
\end{tabular}




\begin{tabular}{|c|c|c|c|c|c|c|c|c|}
\hline methyl vinyl ketone & -2.8 & -39 & 8.9 & -3.2 & -20 & 2.8 & 3.5 & $>3.1^{8}$ \\
\hline $\begin{array}{l}\text { 3,4-dihydroxy-2-butan-2one } \\
\text { (DHBO) }\end{array}$ & -3.4 & -40 & 8.5 & -3.8 & -21 & 2.4 & 3.1 & \\
\hline methacrolein & -5.2 & -38 & 6.1 & -5.6 & -19 & 0.0 & 0.7 & $>3.1^{8}$ \\
\hline $\begin{array}{l}\text { 2,3-dihydroxy-2-methylpropanal } \\
\text { (DHMP) }\end{array}$ & -5.0 & -37 & 6.1 & -5.4 & -18 & 0.0 & 0.7 & \\
\hline 4-hydroxy-2-butanone & -2.8 & -42 & 9.6 & -3.2 & -23 & 3.5 & 4.2 & \\
\hline 4-nitrato-2-butanone & -3.6 & -41 & 8.8 & -4.0 & -22 & 2.6 & 3.4 & \\
\hline 4-sulfato-2-butanone ion & -3.2 & -43 & 9.7 & -3.6 & -24 & 3.6 & 4.3 & \\
\hline 2-pentanone & -2.7 & -44 & 10.3 & -3.1 & -24 & 4.1 & 4.9 & \\
\hline 3-pentanone & -0.4 & -43 & 12.3 & -0.8 & -24 & 6.2 & 6.9 & \\
\hline $\begin{array}{l}\text { 2-hydroxy-2-methyl-4- } \\
\text { sulfato-3-butanone }\end{array}$ & -6.9 & -44 & 6.2 & -7.3 & -25 & 0.1 & 0.8 & \\
\hline $\begin{array}{l}\text { 3-(hydroxymethyl)-2- } \\
\text { methyloxirane-2-carbaldehyde } \\
\text { (1-IEPOXO) }\end{array}$ & -8.1 & -39 & 3.5 & -8.5 & -20 & -2.7 & -1.9 & \\
\hline $\begin{array}{l}\text { 3-(hydroxymethyl)-3- } \\
\text { methyloxirane-2-carbaldehyde } \\
\text { (4-IEPOXO) }\end{array}$ & -8.4 & -34 & 1.8 & -8.8 & -15 & -4.3 & -3.6 & \\
\hline $\begin{array}{l}\text { 2,3,4-trihydroxy-2- } \\
\text { methylbutanal ( } 2-\mathrm{MT}-1- \\
\text { aldehyde) }\end{array}$ & -6.8 & -41 & 5.3 & -7.2 & -21 & -0.9 & -0.1 & \\
\hline $\begin{array}{l}\text { 2,3,4-trihydroxy-3- } \\
\text { methylbutanal (2-MT-4- } \\
\text { aldehyde) }\end{array}$ & -4.9 & -40 & 6.9 & -5.3 & -20 & 0.7 & 1.5 & \\
\hline benzaldehyde & -2.6 & -38 & 8.7 & -3.0 & -19 & 2.5 & 3.3 & \\
\hline
\end{tabular}

Table S1: Computational MG2MS, van't Hoff adjusted, $\Delta_{\mathrm{r}} \mathrm{G}^{\text {exp }}$ adjusted, and experimental thermodynamic values (units: $\Delta_{\mathrm{r}} \mathrm{H}^{\mathrm{o}}$ and $\Delta_{\mathrm{r}} \mathrm{G}^{\mathrm{o}}\left(\mathrm{kcal} \mathrm{mol}^{-1}\right)$ and $\left.\Delta_{\mathrm{r}} \mathrm{S}^{\mathrm{o}}\left(\mathrm{cal} \mathrm{mol}^{-1} \mathrm{~K}^{-1}\right)\right)$. ${ }^{\text {a this work. }}$ 


\section{References}

1. Betterton, E. A.; Hoffmann, M. R., Henry's law constants of some environmentally important aldehydes. Environ. Sci. Technol. 1988, 22, (12), 1415-1418.

2. Sorensen, P. E., The reversible addition of water to glycolaldehyde in aqueous solution. Acta Chem. Scand. 1972, 26, 33573365.

3. Sorensen, P. E.; Bruhn, K.; Lindelov, F., Kinetics and equilibria for the reversible hydration of the aldehyde group in glyoxylic acid. Acta Chem. Scand. 1974, 28a, 162-168.

4. Buschmann, H.-J.; Füldner, H.-H.; Knoche, W., The reversible hydration of carbonyl compounds in aqueous solution. part I, the keto/gem-diol equilibrium. Ber. Bunsenges. Phys. Chem. 1980, 84, (1), 41-44.

5. Nielsen, H.; Sorensen, P. E., Kinetics and equilibria for the solvolysis of lactaldehyde in aqueous solution. Acta Chem. Scand. 1977, 31a, 739-742.

6. Nemet, I.; Vikić-Topić, D.; Varga-Defterdarović, L., Spectroscopic studies of methylglyoxal in water and dimethylsulfoxide. Bioorg. Chem. 2004, 32, (6), 560-570.

7. Wiberg, K. B.; Morgan, K. M.; Maltz, H., Thermochemistry of carbonyl reactions. 6. a study of hydration equilibria. J. Am. Chem. Soc. 1994, 116, (24), 11067-11077.

8. Zhao, R.; Lee, A. K. Y.; Soong, R.; Simpson, A. J.; Abbatt, J. P. D., Formation of aqueous-phase $\alpha$-hydroxyhydroperoxides ( $\alpha$ HHP): potential atmospheric impacts. Atmos. Chem. Phys. 2013, 13, (12), 5857-5872. 